\title{
Known Interferent
}

National Cancer Institute

\section{Source}

National Cancer Institute. Known Interferent. NCI Thesaurus. Code C139522.

Known interferent in the sample identified. 